
\title{
S Research Square \\ Diagnostic and Immunotherapeutic Value of CD248 in Renal Cell Carcinoma
}

\section{Keying Zhang}

Department of Urology, Xijing Hospital, Fourth Military Medical University, Xi'an 710032, China

\section{Yao Jiang}

Department of Urology, Xijing Hospital, Fourth Military Medical University, Xi'an 710032, China

\section{Xiaolong Zhao}

Department of Urology, Xijing Hospital, Fourth Military Medical University, Xi'an 710032, China

\section{Shaojie Liu}

Department of Urology, Xijing Hospital, Fourth Military Medical University, Xi'an 710032, China

\section{Chao Xu}

Department of Urology, Xijing Hospital, Fourth Military Medical University, Xi'an 710032, China

\section{Shanjin Ma}

Department of Urology, Tangdu Hospital, Fourth Military Medical University, Xi'an 710032, China

\section{Fa Yang}

Department of Urology, Xijing Hospital, Fourth Military Medical University, Xi'an 710032, China

\section{Yan Wang}

Department of Emergency, 987th Hospital, PLA, Baoji, 721000, China

\section{Ping Meng}

Department of Urology, Xijing Hospital, Fourth Military Medical University, Xi'an 710032, China

\section{Changhong Shi}

Laboratory Animal Center, Fourth Military Medical University, Xi'an 710032, China

\section{Donghui Han}

Department of Urology, Xijing Hospital, Fourth Military Medical University, Xi'an 710032, China

\section{Weihong Wen}

Institute of Medical Research, Northwestern Polytechnical University, Xi'an 710072, China

\section{Weijun Qin ( $\nabla$ qinwj@fmmu.edu.cn )}

Fourth Military Medical University

\section{Research}

Keywords: Renal cell carcinoma, CD248, Immunotherapy, Prognosis, Underlying mechanism

Posted Date: November 19th, 2020 
DOI: https://doi.org/10.21203/rs.3.rs-108745/v1

License: (c) (1) This work is licensed under a Creative Commons Attribution 4.0 International License. Read Full License 


\section{Abstract}

Background: Renal cell carcinoma (RCC) is the most common malignancy in urinary system. Despite substantial improvements in available treatments, survival outcome of advanced RCC is unsatisfied. Identifying novel biomarker to assist early diagnosis and screen immunotherapy sensitive patients would be beneficial. CD248 is a promising candidate that deserves to be investigated.

Methods: The Cancer Genome Atlas (TCGA) dataset and clinical specimen were adopted to analyze CD248 expression between normal and tumor tissues. Univariate and multivariate Cox regression analysis was employed to identify independent prognostic factors and construct a CD248-based prognostic signature. The correlation among present signature, tumor infiltrating immune cells, tumor mutation burden (TMB), and immunomodulatory molecules were evaluated. Weighted gene coexpression network analysis (WGCNA) and enrichment analysis was performed to explore the underlying mechanism of CD248 in RCC progression.

Results: CD248 overexpressed in RCC was related with a poor prognosis, and CD248-based prognostic signature could precisely stratify RCC patients with different survival outcomes regardless of training or testing cohort. Present signature could reflect immunosuppressive landscape of RCC (i.e. increased regulatory $T$ cells infiltration and upregulated immune checkpoints), which accompanied with deteriorated clinicopathologic indexes. TMB and immunostimulatory molecules expression also increased with the risk score generated from present signature. CD248 co-expressed gene sets were identified through WGCNA algorithm, and several immunosuppressive GO terms and KEGG pathways were significantly enriched.

Conclusion: CD248 is a valuable biomarker to improve diagnostic and therapeutic efficiency of RCC. Immunosuppressive effect of CD248 co-expressed genes may provide insight for present study.

\section{Background}

Renal cell carcinoma (RCC) is the seventh most common neoplasm in developed world and the most lethal malignancy in urinary system [1]. As reported, RCC morbidity has more than doubled in North America and Western Europe over the past half-century, which is predicted to rapidly increase in Latin America, Asia and Africa in the coming decades [2]. Actually, RCC is an insidious neoplasm with one-third cases initially diagnosed as metastatic, which survival rate is abysmally low. Despite treatments for RCC have been revolutionized by targeted therapy, 5-year survival rate of advanced/metastatic RCC is only $12 \%$ [3]. Immunotherapy with immune checkpoint inhibitors to block PD1, CTLA4, and LAG3 is another promising method to promote survival outcome of RCC patients [4-6]. However, low response rate restricts its therapeutic efficacy [7]. Hence, identify novel biomarker to facilitate earlier diagnosis of asymptomatic patient and assist clinician to screen the one who is sensitive to immunotherapy would be beneficial for RCC prognosis. 
Tumor endothelial markers (TEMs) involved with tumor specific angiogenesis play a crucial role in tumor development and progression, among which TEM1 (also known as endosialin or CD248) is specifically overexpressed in tumor-associated fibroblasts and pericytes residing in the tumor blood vessels. It has been found that CD248 is an essential molecular associated with cell adhesion, migration, and stromal cell proliferation [8]. Once CD248 knockout in mice, there was a striking reduction in tumor growth, invasiveness, and metastasis after tumor transplantation, indicating that CD248-positive stroma would promote malignancy [9]. Based on the important regulatory mechanism of CD248 in supporting tumor stromal cell function and tumor growth, CD248 is regarded as an indicator to evaluate tumor grade and prognosis [10], as well as a potent target to disrupt tumor vasculature and control tumor growth [11]. However, whether CD248 can predict RCC prognosis and guide immunotherapy is largely unknown.

To explore the predictive value of CD248 in RCC, we performed present study. The Cancer Genome Atlas (TCGA) dataset and clinical specimen were adopted to analyze CD248 expression difference between normal and tumor tissues. Then, we constructed a CD248-based prognostic signature by integrating multiple clinical variables, which acquired promoted predictive accuracy. The correlation among present signature, tumor infiltrating immune cells (TIICs), tumor mutation burden (TMB), and immunomodulatory molecules were also evaluated. Finally, weighted gene co-expression network analysis (WGCNA) and enrichment analysis were performed to explore the underlying mechanism of CD248 in RCC progression.

\section{Methods}

\section{Data source and preprocessing}

895 RCC data and 128 non-tumor data were downloaded from TCGA portal (https://portal.gdc.cancer.gov/). Transcriptomic data (RNA-Seq FPKM) and clinical information were integrated through ID numbers. Replicate gene probes were replaced with their average via limma package [12]. All data were processed and analyzed with R software (https://www.r-project.org/).

\section{Differential expression analysis of CD248}

Differentially expressed genes (DEGs) between tumor and normal tissue were analyzed through Wilcox test. $P$-value was adjusted with false discovery rate (FDR), and filter criteria was FDR $<0.05$ and $\| \log _{2}$ fold-change $[\mathrm{FC}] \mid>1$. The expression of CD248 between groups was analyzed through $t$-test, and visualized with GraphPad Prism 8 (GraphPad Software, San Diego, CA, USA). A body-map of CD248 expression was obtained from Gene Expression Profiling Interactive Analysis (GEPIA) website (http://gepia.cancer-pku.cn/), and the expression median was normalized to transcripts per million (TPM).

\section{Validation of CD248 expression in clinical specimen}

Paraffin-embedded tissue microarrays (Outdo Biotech, Shanghai, China) were deparaffinized, rehydrated, and treated with $3 \%$ hydrogen peroxide for 10 min to inhibit endogenous peroxidase activity. Heat 
mediated antigen retrieval was performed in citrate buffer for 2 min. After being blocked with $5 \%$ bovine serum albumin (BSA) for 30 min, slides were incubated with rabbit anti-human CD248 primary antibody (1:2000, ab204914, Abcam, MA, USA) overnight at $4^{\circ} \mathrm{C}$. Immunodetection was performed using the standard rapid EnVision technique (Dako, Glostrup, Denmark). Subsequently, slides were washed in distilled water, and counterstained with hematoxylin. Digital images were obtained using an optical microscope (BX51, Olympus, Tokyo, Japan).

\section{Prognostic value analysis of CD248 in RCC}

TCGA-RCC patients were divided into high-expression and low-expression group according to the median expression level of CD248. Then, Kaplan-Meier survival analysis and receiver operating characteristic (ROC) analysis were performed to evaluate the prognostic value and predictive accuracy of CD248, respectively. Univariate and multivariate Cox regression analysis were employed to identify the independent prognostic factors of RCC. $P<0.05$ was considered statistically significant.

\section{Construction and validation of CD248-based prognostic signature}

TCGA-RCC patients with complete clinical information $(n=246)$ were used as a training cohort, and TCGA-clear cell RCC (cCRCC) patients with certain clinical information (i.e. age, histological grade, pathological stage and M status) were selected as a testing cohort $(n=489)$. Training cohort was used to construct a CD248-based prognostic signature, and testing cohort was used to confirm its performance. Multivariate Cox regression analysis was adopted to construct present signature by integrating clinicopathological variables (i.e. age, gender, histological grade, pathological stage, and TNM status) with CD248 expression level. Risk score of each patient was calculated using the following formula and the median was employed to separate both cohorts into different risk groups [13]:

\section{Risk score $=\sum_{i=1}^{n}$ coefficient (gene i) $*$ Expression value of (gene i)}

Survival and ROC analysis were performed as aforementioned. To visualize present signature, a nomogram was constructed by rms R package.

\section{Correlation between CD248-based signature and TIICs}

Tumor purity and immune score of TCGA-RCC patients were assessed through ESTIMATE R package as previously reported [14]. Relative fraction of 22 TIICs types in each sample was quantified by CIBERSORT method and LM22 signature matrix $[15,16]$. The algorithm ran at 100 permutations with a threshold of $P$ $<0.05$ to select eligible patients [17]. The correlation between risk score and TIICs was analyzed with Pearson correlation coefficient test, and the impact of TIICs on clinicopathological features was analyzed with Wilcox test and Kruskal-Wallis test. The box plot was prepared with beeswarm $\mathrm{R}$ package. 


\section{Correlation between CD248-based signature and TMB}

Masked somatic mutation data (VarScan) of RCC were retrieved from TCGA portal. Non-synonymous somatic mutations of each patient were counted by Perl software (https://www.perl.org/). Then, we used $38 \mathrm{Mb}$ as the estimate of exome size, and calculated TMB (i.e. mutation density) with the following formula: $T M B=$ Total mutation frequency/38. Wilcox test was adopted to evaluate the relationship between TMB and risk score or clinicopathological features. $P<0.05$ was considered statistically significant.

\section{Correlation between CD248-based signature and immunomodulatory molecules}

Immune checkpoint molecules (i.e. PD1, CTLA4, LAG3, TIM3, BTLA, and VSIR) and immunostimulatory molecules (i.e. CD28, CD27, TNFRSF4, TNFRSF9, and TNFRSF18) play important roles in immunoregulation. In present study, the expression level of aforementioned molecules between two risk groups was analyzed with Wilcox test. Kaplan-Meier survival analysis was performed using R software, and median expression level was used as cut-off value. $P<0.05$ was considered statistically significant.

\section{WGCNA and enrichment analysis}

DEGs co-expressed with CD248 were selected through Pearson correlation coefficient test and visualized with pheatmap R package. Filter criteria was |correlation coefficient $\mid>0.5$ and $P<0.001$. WGCNA algorithm was employed to identify CD248 co-expressed modules. Briefly, gradient method was used to screen out the appropriate power value with independence degree of 0.9. Cluster analysis was performed to construct a dendrogram, and module-trait heatmap was painted to identify the phenotype (clinic trait) highly correlated expression set (module). Finally, the interested modules were visualized with Cytoscape 3.6.0 and analyzed with GO and KEGG enrichment analysis. FDR $<0.05$ was used as the threshold.

\section{Results}

\section{CD248 overexpressed in RCC tissues}

3086 DEGs were obtained based on TCGA-RCC dataset, among which 1127 genes were downregulated, and 1959 genes were upregulated in RCC tissues compared with the normal $\left(F D R<0.05, \| \log _{2} F C \mid>1\right.$, Fig. 1a and b). Then, CD248 overexpressed in RCC tissues was identified ( $P<0.0001$, Fig. 1c). Body-map of CD248 showed that the median expression level in RCC was 4.94, which was much higher than 3.28 in normal kidney (Fig. 1d). Immunohistochemical staining results indicated that CD248 expression was upregulated in RCC instead of adjacent normal tissue (Fig. 1e). As shown in Fig. 1f, the prognosis was poorer in high-CD248 expression group than that in low-expression group $(P<0.0001)$. Precisely, the overall survival (OS) rate at 5-year for high-expression group was $58.8 \%$, and the corresponding rate for low-expression group was $75.3 \%$. The area under ROC curve (AUC) was 0.662 , suggesting that CD248 could accurately predict the OS of RCC patients (Fig. 1g). Additionally, univariate and multivariate Cox 
regression analysis revealed that CD248 could serve as an independent prognostic factor for RCC $(P<$ 0.05 , Fig. $1 \mathrm{~h}$ and i).

\section{Prognostic value of CD248-based signature}

Training cohort was adopted to construct a CD248-based signature. After deleting clinicopathological variables that would overfit the signature, coefficients were estimated through multivariate Cox regression. Subsequently, the risk score for each patient was calculated with the following formula:

Risk score $=(0.0291 \times$ Age $)+(0.4245 \times$ Histological grade $)+(0.3303 \times$ Pathological stage $)+(0.6492 \times$ $\mathrm{M}$ status $)+(0.0038 \times \mathrm{CD} 248$ expression level $)$

According to the median risk score 0.8618 , individuals in the training cohort were sorted into a high-risk group $(n=123)$ and a low-risk group $(n=123)$. Kaplan-Meier survival analysis showed that the prognosis was worse in high-risk group than that in low-risk group $(P<0.0001$, Fig. 2a). The OS rate at 5-year for high-risk group and low-risk group was $31.7 \%$ and $77.7 \%$, respectively. Then, we ranked patients with risk score and analyzed their survival status. As shown in Fig. 2b, a large amount of death distributed in highrisk group. The AUC value for present signature was 0.889 (Fig. 2c). To facilitate clinical utility, a nomogram to predict RCC prognosis at 3-, 5- and 10-year was prepared accordingly (Fig. 2d).

Testing cohort was used to verify the accuracy of present signature. As shown in Fig. 2e and $\mathrm{f}$, survival status of ccRCC patients differed significantly between two risk groups $(P<0.05)$. The survival rates at 3and 5 -year in high-risk group were $58.1 \%$ and $44.4 \%$, respectively, while the corresponding rates in low-risk group were $92.1 \%$ and $78.0 \%$, respectively. Moreover, the AUC value of present signature was 0.801 in testing cohort (Fig. 2g).

\section{Correlation between present signature and tumor immune microenvironment}

ESTIMATE and CIBERSORT algorithm were employed to assess tumor purity and infiltrating immune cells (Fig. 3a and d). As shown in Fig. 3b and c, increased immune score was related with deteriorated histological grade and pathological stage $(P<0.05)$. The fraction of $\operatorname{CD} 8^{+} T$ cells and regulatory $T$ cells (Tregs) were positively related with the risk score generated by present signature $(P<0.05$, Fig. $3 e$ and $\mathrm{f})$. Furthermore, a high proportion of $\mathrm{CD}^{+} \mathrm{T}$ cells and Tregs could lead to a poor prognosis of RCC patients, accompanied with increased histological grade, bad pathological stage, and detrimental TNM status $(P<$ 0.05 , Fig. 3g-k).

TMB is a vital factor affecting tumor immune response and immunotherapy. In present study, with the increase of risk score generated by present signature, TMB significantly increased $(P<0.05$, Fig. 31$)$. Besides, higher TMB was associated with worse histological grade, and the value of TMB was increased with patients' age $(P<0.05$, Fig. $3 \mathrm{~m}$ and $\mathrm{n})$. 
The expression level of immunomodulatory molecules was regarded as a promising indicator to guide immunotherapy. We found that the expression of some immune checkpoint molecules (i.e. PD1, CTLA4, and LAG3) were increased in high-risk group $(P<0.01$, Fig. 4a-c), while others (i.e. TIM3, BTLA, and VSIR) were unchanged between two risk groups $(P>0.05$, Fig. 4d-f). Survival analysis indicated that highlyexpressed PD1 companied with a bad prognosis $(P<0.0001$, Fig. $4 \mathrm{~g})$, while expression level of CTLA4 and LAG3 did not significantly affect the OS of RCC patients ( $P>0.05$, Fig. $4 \mathrm{~h}$ and $\mathrm{i})$. In addition to immune checkpoint, the expression of immunostimulatory molecules has been investigated as well. As shown in Fig. 5, the expression of commonly detected immunostimulatory molecules, such as CD28, CD27, TNFRSF4, TNFRSF9, and TNFRSF18, were upregulated in high-risk group $(P<0.05)$.

\section{WGCNA and enrichment analysis of CD248 co-expressed genes}

334 DEGs co-expressed with CD248 were selected through Pearson correlation coefficient test (|correlation coefficient $\mid>0.5$ and $P<0.001$ ). The top $15 \mathrm{DEGs}$ that positively and negatively correlated with CD248 were adopted to develop a co-expressed heatmap (Fig. 6a). Subsequently, we identified five distinct CD248 co-expressed modules through WGCNA algorithm (Fig. 6b). Module-trait heatmap indicated that blue and turquoise module were significantly associated with RCC progression $(P<0.05$, Fig. 6c). Then, intramodular and extramodular interactions were visualized, especially the module marked with blue and turquoise (Fig. 6d, e and h). With the co-expressed network, several hub genes with maximum intramodular connectivity were identified, which might play a vital role in RCC progression.

To explore the underlying mechanism of blue and turquoise module on RCC progression, GO and KEGG enrichment analysis were performed. As shown in Fig. $6 \mathrm{f}$ and i, "Negative regulation of defense response", "Negative regulation of immune system process", "Negative regulation of leukocyte activation", "Negative regulation of cell adhesion" etc. immunosuppressive GO terms were significantly enriched (FDR < 0.05). Then, KEGG enrichment analysis showed that several immunomodulatory signaling pathways were significantly enriched, including "PI3K-Akt signaling pathway", "Cytokinecytokine receptor interaction", "Th1 and Th2 cell differentiation", "Natural killer cell mediated cytotoxicity", and "NOD-like receptor signaling pathway" (FDR $<0.05$, Fig. $6 \mathrm{~g}$ and j).

\section{Discussion}

RCC incidence has increased steadily by about $1 \%$ per year, and the high mortality rate remains unchanged worldwide [18]. Despite substantial improvements in available treatment options, 5-year survival rate for advanced/metastatic RCC is less than $23 \%$ [19]. In fact, about $30-50 \%$ RCC patients have missed the best surgical opportunity due to the occult incidence [20]. Immunotherapy is an emerging method to prolong the OS of RCC. However, low response rate results in an unsatisfied clinical outcome. Accordingly, various biomarkers have been suggested to assist early diagnosis and guide treatment selection. Chen et al. reported that miR-30a-3p could inhibit RCC invasion and serve as a new prognostic marker [21]. miR-142-3p has also proved to be involved with tumorigenesis and RCC development [22]. In addition to miRNAs, mRNAs including HHLA2 and syntaxin 6 were associated with decreased survival, 
and corresponding inhibitors held promise as novel therapy against RCC [23, 24]. However, the functional effect of single gene in RCC progression is relatively weak. Identifying sensitive and specific indicator to improve diagnostic and therapeutic efficiency is still urgently needed.

Recently, a great amount of evidence indicates that TEMs have broad influence in complicated crosstalk between tumor cells and tumor microenvironment, which would lead to tumor progression $[25,26]$. Thus, TEMs appear to be promising candidates for tumor early detection, monitoring, and treatment. As an important part of TEMs, the biological function of CD248 in RCC remains unclear. In present study, the expression level of CD248 in RCC tissues was evaluated through TCGA dataset and confirmed in external clinical specimens. We found the expression of CD248 significantly upregulated in RCC, compared with adjacent normal kidney tissues. Besides, highly-expressed CD248 was associated with bad prognosis. CD248 could also serve as an independent prognostic factor to predict RCC patients' OS, and the predictive accuracy (AUC $=0.662$ ) was regarded as acceptable [27]. These finding above indicates that CD248 expression level could be a new early diagnostic and prognostic marker for RCC. Subsequently, to establish a clinically stratifying system to improve diagnostic efficiency, we constructed a CD248-based prognostic signature. This signature could stratify TCGA-RCC patients into two risk groups with statistically different survival outcomes, and the predictive accuracy (AUC $=0.889$ ) was deemed to be excellent [27]. The reliability of present signature was further verified in a testing cohort and a nomogram was prepared to facilitate its clinical application.

Tumor immune microenvironment comprised with infiltrating immune cells and immune-related proteins (IRPs) has emerged as an important player in tumor progression [28, 29]. In present study, we explored the correlation between present signature and dysfunctional immune microenvironment. We found that Tregs infiltration in RCC significantly increased with the risk score generated by present signature, and high immune score as well as high Tregs infiltration accompanied with bad histological grade, advanced pathological stage, and more chance of metastasis, which consisted with previous study [30].

Additionally, $\mathrm{CD}^{+}$cytotoxic T lymphocytes (CTLs) were positively correlated with the risk score, however, increased CTLs resulted in RCC metastasis instead of killing effect. Thus, we speculate CTLs-mediated anti-tumor response is counterbalanced by strong immunosuppression of Tregs, which consequentially facilitate the survival of cancer cells [31, 32].

The killing efficacy of CTLs is also directly or indirectly regulated by IRPs and TMB [33-35]. The immune checkpoints (i.e. PD1, CTLA4, and LAG3) were upregulated in high-risk group, which might induce CTLs depletion, tumorimmuneescape, and poor survival outcome. As well, commonly detected immunostimulatory molecules (i.e. CD28, CD27, TNFRSF4, TNFRSF9, and TNFRSF18) were upregulated in high-risk group. TMB-a surrogate for neoantigen level and malignant degree-was increased with risk score generated by present signature. Based on the immune landscape of high-risk patients, once immune suppression of CTLs is removed, the self-anti-tumor immune response would be expanded and high-risk patients might benefit from immunotherapy. Therefore, present signature could not only contribute to RCC patient's early diagnosis, but also assist clinician to screen immunotherapeutic 
sensitive patients. Inevitably, large-scale prospective validation of clinical benefit before widespread adoption are necessary.

To explore possible functions of CD248 in RCC, WGCNA algorithm and enrichment analysis were performed. The results suggested that CD248 co-expressed genes could be divided into five modules, among which blue and turquoise module were significantly associated with RCC progression. Then, the identified prognostic-related modules were analyzed with GO and KEGG algorithm. As a result, several immunosuppressive $\mathrm{GO}$ terms were significantly enriched, including negative regulation of leukocyte activation, migration, adhesion and differentiation, which might provide insight for CTLs depletion mentioned above. Besides, several immunomodulatory signaling pathways and intramodular hub genes were identified. After verifying them in vitro or in vivo studies, novel diagnostic and therapeutic targets might be proposed.

\section{Conclusions}

We identified a valuable biomarker and constructed a reliable prognostic signature that can precisely predict the prognosis of RCC patients. Additionally, present signature can effectively screen out RCC patients suitable for immunotherapy. WGCNA and enrichment analysis revealed possible functions of CD248 and co-expressed genes, which may contribute to explain CD248-mediated RCC progression and provide potential diagnostic and therapeutic targets.

\section{Abbreviations}

AUC: Area under ROC curve; BSA: Bovine serum albumin; ccRCC: Clear cell RCC; CTLs: Cytotoxic T lymphocyte; DEGs: Differentially expressed genes; FDR: False discovery rate; GEPIA: Gene Expression Profiling Interactive Analysis; IRPs: Immune-related proteins; OS: Overall survival; RCC: Renal cell carcinoma; ROC: Receiver operating characteristic; TCGA: The Cancer Genome Atlas; TEMs: Tumor endothelial markers; TIICs: Tumor infiltrating immune cells; TMB: Tumor mutation burden; TPM: Transcripts per million; Tregs: Regulatory T cells; WGCNA: Weighted gene co-expression network analysis.

\section{Declarations}

\section{Acknowledgements}

Not applicable.

\section{Authors' contributions}

$\mathrm{KYZ}, \mathrm{YJ}$, and $\mathrm{XLZ}$ : design, analysis and interpretation of data, drafting of the manuscript, critical revision of the manuscript; KYZ, YJ, XLZ, SJL, CX, and SJM: statistical analysis; FY, YW, PM, and CHS: data acquisition and literature search; DHH, WHW and WJQ: critical revision of the manuscript, administrative support, obtaining funding, supervision. All authors read and approved the final manuscript. 


\section{Funding}

The work was supported by grants from the National Natural Science Foundation of China (No. 81772734, 81802935) and the Innovation Capability Support Plan of Shaanxi Province (2020PT-021). The funders had no role in the study design, data collection, data analysis, interpretation, and report writing.

\section{Availability of data and materials}

The datasets analyzed during the current study are available in the TCGA repository (https://portal.gdc.cancer.gov).

\section{Ethics approval and consent to participate}

Not applicable

\section{Consent for publication}

Not applicable

\section{Competing interests}

The authors declare that they have no competing interests.

\section{References}

1. Siegel RL, Miller KD, Jemal A. Cancer statistics, 2019. CA Cancer J Clin. 2019;69:7-34.

2. Padala SA, Barsouk A, Thandra KC, Saginala K, Mohammed A, Vakiti A, et al. Epidemiology of Renal Cell Carcinoma. World J Oncol. 2020;11:79-87.

3. Miller KD, Nogueira L, Mariotto AB, Rowland JH, Yabroff KR, Alfano CM, et al. Cancer treatment and survivorship statistics, 2019. CA Cancer J Clin. 2019;69:363-85.

4. Peinemann F, Unverzagt S, Hadjinicolaou AV, Moldenhauer I. Immunotherapy for metastatic renal cell carcinoma: A systematic review. J Evid Based Med. 2019;12:253-62.

5. Topalian SL, Hodi FS, Brahmer JR, Gettinger SN, Smith DC, McDermott DF, et al. Five-Year Survival and Correlates Among Patients with Advanced Melanoma, Renal Cell Carcinoma, or Non-Small Cell Lung Cancer Treated with Nivolumab. JAMA ONCOL. 2019;5:1411-20.

6. Rini BI, Pal SK, Escudier BJ, Atkins MB, Hutson TE, Porta C, et al. Tivozanib versus sorafenib in patients with advanced renal cell carcinoma (TIVO-3): a phase 3, multicentre, randomised, controlled, open-label study. LANCET ONCOL. 2020;21:95-104.

7. Motzer RJ, Escudier B, McDermott DF, George S, Hammers HJ, Srinivas S, et al. Nivolumab versus Everolimus in Advanced Renal-Cell Carcinoma. N Engl J Med. 2015;373:1803-13. 
8. Tomkowicz B, Rybinski K, Foley B, Ebel W, Kline B, Routhier E, et al. Interaction of endosialin/TEM1 with extracellular matrix proteins mediates cell adhesion and migration. Proc Natl Acad Sci U S A. 2007;104:17965-70.

9. Nanda A, Karim B, Peng Z, Liu G, Qiu W, Gan C, et al. Tumor endothelial marker 1 (Tem1) functions in the growth and progression of abdominal tumors. Proc Natl Acad Sci U S A. 2006;103:3351-6.

10. Hong YK, Lee YC, Cheng TL, Lai CH, Hsu CK, Kuo CH, et al. Tumor Endothelial Marker 1 (TEM1/Endosialin/CD248) Enhances Wound Healing by Interacting with Platelet-Derived Growth Factor Receptors. J INVEST DERMATOL. 2019;139:2204-14.

11. Facciponte JG, Ugel S, De Sanctis F, Li C, Wang L, Nair G, et al. Tumor endothelial marker 1-specific DNA vaccination targets tumor vasculature. J CLIN INVEST. 2014;124:1497-511.

12. Yue $\mathrm{C}, \mathrm{Ma} \mathrm{H}$, Zhou Y. Identification of prognostic gene signature associated with microenvironment of lung adenocarcinoma. PEERJ. 2019;7:e8128-44.

13. Wan B, Liu B, Huang Y, Yu G, Lv C. Prognostic value of immune-related genes in clear cell renal cell carcinoma. Aging (Albany NY). 2019;11:11474-89.

14. Yoshihara K, Shahmoradgoli M, Martínez E, Vegesna R, Kim H, Torres-Garcia W, et al. Inferring tumour purity and stromal and immune cell admixture from expression data. NAT COMMUN. 2013;4:2612.

15. Newman AM, Liu CL, Green MR, Gentles AJ, Feng W, Xu Y, et al. Robust enumeration of cell subsets from tissue expression profiles. NAT METHODS. 2015;12:453-7.

16. Zeng D, Zhou R, Yu Y, Luo Y, Zhang J, Sun H, et al. Gene expression profiles for a prognostic immunoscore in gastric cancer. Br J Surg. 2018;105:1338-48.

17. Ali HR, Chlon L, Pharoah PD, Markowetz F, Caldas C. Patterns of Immune Infiltration in Breast Cancer and Their Clinical Implications: A Gene-Expression-Based Retrospective Study. PLOS MED. 2016;13:e1002194-218.

18. Zhu WK, Xu WH, Wang J, Huang YQ, Abudurexiti M, Qu YY, et al. Decreased SPTLC1 expression predicts worse outcomes in cCRCC patients. J CELL BIOCHEM. 2020;121:1552-62.

19. Motzer RJ, Jonasch E, Agarwal N, Bhayani S, Bro WP, Chang SS, et al. Kidney Cancer, Version 2.2017, NCCN Clinical Practice Guidelines in Oncology. J Natl Compr Canc Netw. 2017;15:804-34.

20. Qin S, Shi X, Wang C, Jin P, Ma F. Transcription Factor and miRNA Interplays Can Manifest the Survival of ccRCC Patients. Cancers (Basel). 2019;11.

21. Chen Y, Zhou J, Wu X, Huang J, Chen W, Liu D, et al. miR-30a-3p inhibits renal cancer cell invasion and metastasis through targeting ATG12. Transl Androl Urol. 2020;9:646-53.

22. Peng X, Pan X, Liu K, Zhang C, Zhao L, Li H, et al. miR-142-3p as a novel biomarker for predicting poor prognosis in renal cell carcinoma patients after surgery. Int J Biol Markers. 2019;34:302-8.

23. Chen L, Zhu D, Feng J, Zhou Y, Wang Q, Feng H, et al. Overexpression of HHLA2 in human clear cell renal cell carcinoma is significantly associated with poor survival of the patients. CANCER CELL INT. 2019;19:101. 
24. Peak TC, Su Y, Chapple AG, Chyr J, Deep G. Syntaxin 6: A novel predictive and prognostic biomarker in papillary renal cell carcinoma. SCI REP-UK. 2019;9:3146.

25. Pietrzyk $Ł$. Biomarkers Discovery for Colorectal Cancer: A Review on Tumor Endothelial Markers as Perspective Candidates. DIS MARKERS. 2016;2016:4912405.

26. Wang X, Zhu Q, Lin Y, Wu L, Wu X, Wang K, et al. Crosstalk between TEMs and endothelial cells modulates angiogenesis and metastasis via IGF1-IGF1R signalling in epithelial ovarian cancer. $\mathrm{Br} J$ Cancer. 2017;117:1371-82.

27. Cho SH, Pak K, Jeong DC, Han ME, Oh SO, Kim YH. The AP2M1 gene expression is a promising biomarker for predicting survival of patients with hepatocellular carcinoma. J CELL BIOCHEM. 2019;120:4140-6.

28. Sobral-Leite M, Salomon I, Opdam M, Kruger DT, Kok M. Cancer-immune interactions in ER-positive breast cancers: PI3K pathway alterations and tumor-infiltrating lymphocytes. Breast cancer research: BCR. 2019;21:90-102.

29. McGranahan N, Furness AJ, Rosenthal R, Ramskov S, Lyngaa R, Saini SK, et al. Clonal neoantigens elicit T cell immunoreactivity and sensitivity to immune checkpoint blockade. SCIENCE. 2016;351:1463-9.

30. Wang Y, Yang J, Zhang Q, Xia J, Wang Z. Extent and characteristics of immune infiltration in clear cell renal cell carcinoma and the prognostic value. Transl Androl Urol. 2019;8:609-18.

31. Pol JG, Caudana P, Paillet J, Piaggio E, Kroemer G. Effects of interleukin-2 in immunostimulation and immunosuppression. J EXP MED. 2020;217.

32. Pai SI, Marincola FM. Peripheral Circulating CD45RA(-)FOXP3(hi) T Regulatory (T(R)(eg)) II Cells Provide a Window into the Activity of Intratumoral T(R)(eg) Cells. Trends Cancer. 2020;6:3-6.

33. Mitchell TJ, Rossi SH, Klatte T, Stewart GD. Genomics and clinical correlates of renal cell carcinoma. WORLD J UROL. 2018;36:1899-911.

34. Cheng W, Ren X, Zhang C, Cai J, Liu Y, Han S, et al. Bioinformatic profiling identifies an immunerelated risk signature for glioblastoma. NEUROLOGY. 2016;86:2226-34.

35. Dudani S, Savard MF, Heng D. An Update on Predictive Biomarkers in Metastatic Renal Cell Carcinoma. Eur Urol Focus. 2020;6:34-6.

\section{Figures}


a

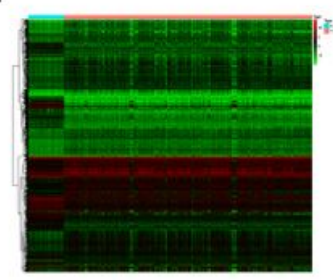

b

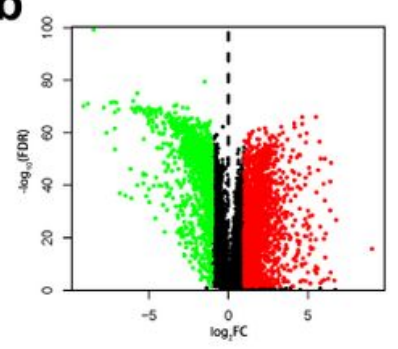

C

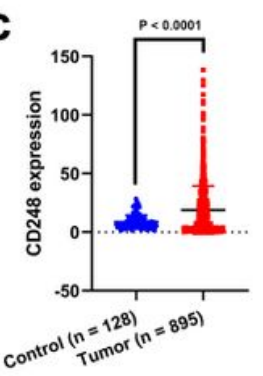

d

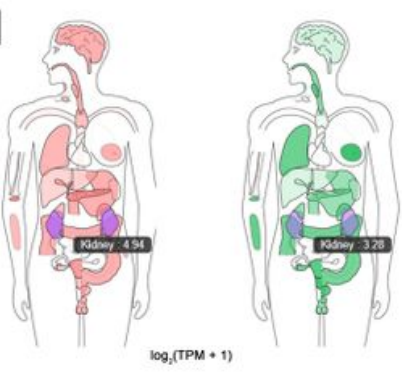

e
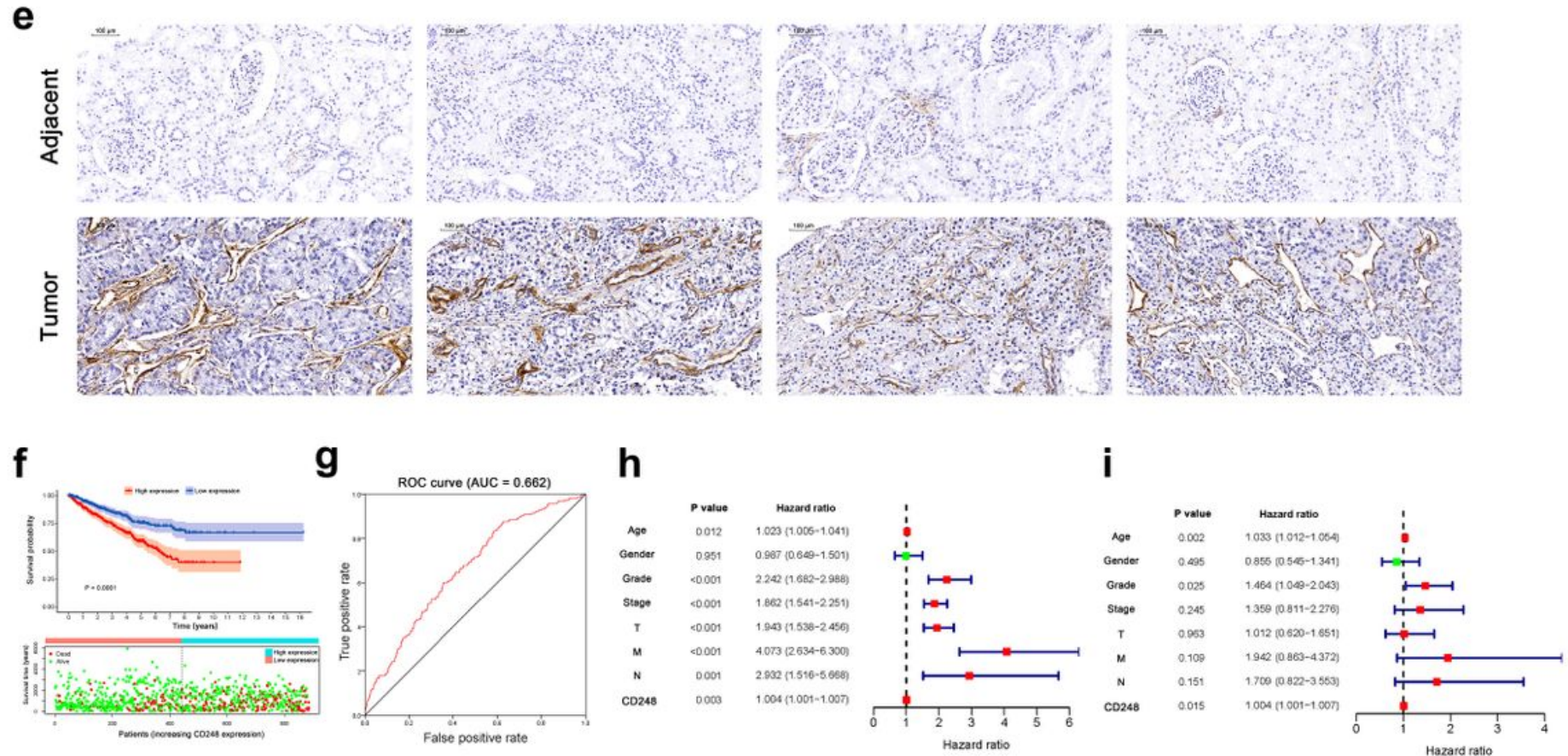

\section{Figure 1}

Expression and prognostic value of CD248. (a) Heat map of DEGs. Green to red spectrum indicates low to high gene expression. (b) Volcano plot of DEGs. Red, green and black dots represent upregulated, downregulated and unchanged genes, respectively. (c) CD248 overexpressed in RCC tissues. (d) Bodymap of CD248 expression. (e) Immunohistochemical staining of CD248. Scale bar $=100 \mu \mathrm{m}$. (f) Prognostic value of CD248. 95\% confidence interval is shown as light-colored background. (g) ROC curve of CD248. (h) Univariate Cox regression analysis of CD248. (i) Multivariate Cox regression analysis of CD248. Red and green dots represent variables with hazard ratio $>1$ and $\leq 1$, respectively. $P<0.05$ was considered statistically significant. 
a

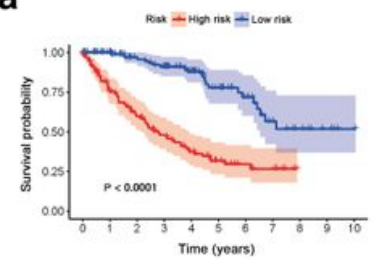

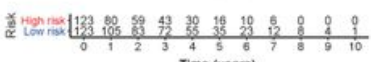

b
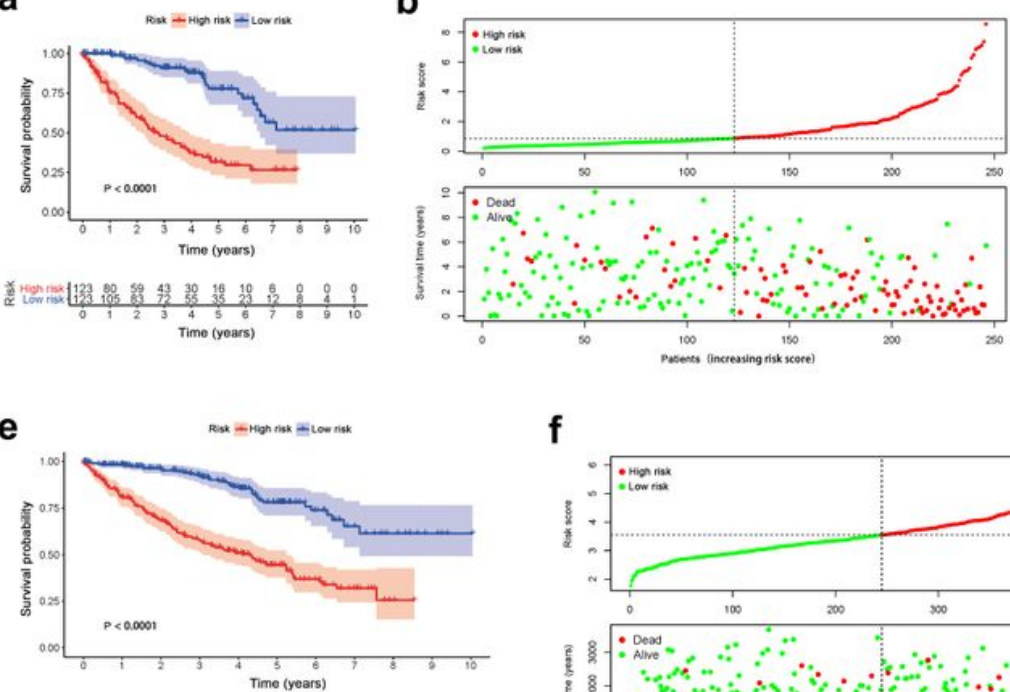

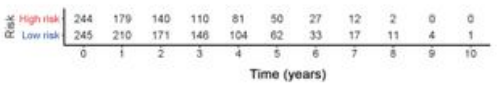

$\mathbf{f}$
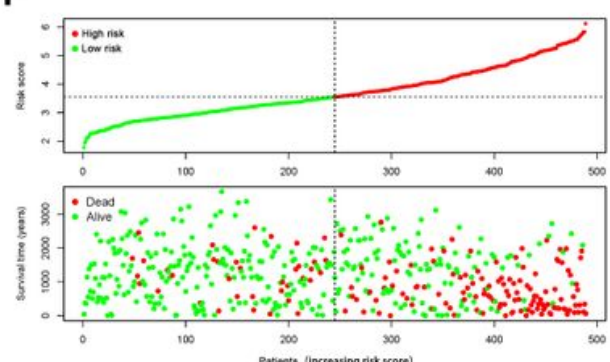

C

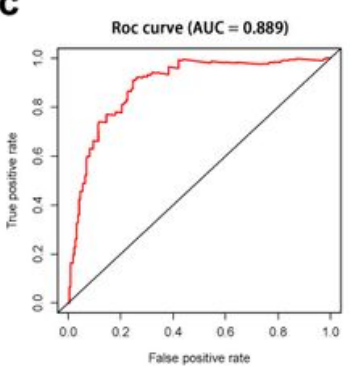

d

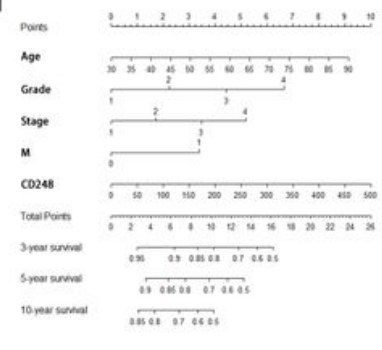

g

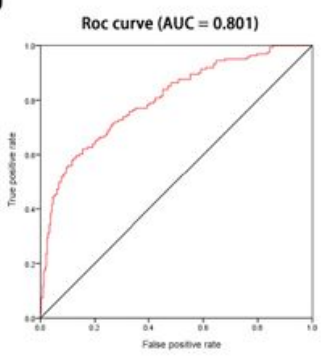

Figure 2

Prognostic value of CD248-based signature. (a) Kaplan-Meier curve of training cohort. (b) Distribution of risk score and survival status of each patient in training cohort. (c) ROC curve of present signature in training cohort. (d) Nomogram of present signature. (e) Kaplan-Meier curve of testing cohort. (f) Distribution of risk score and survival status of each patient in testing cohort. $(\mathrm{g})$ ROC curve of present signature in testing cohort. 95\% confidence interval is shown as light-colored background. $\mathrm{P}<0.05$ was considered statistically significant.
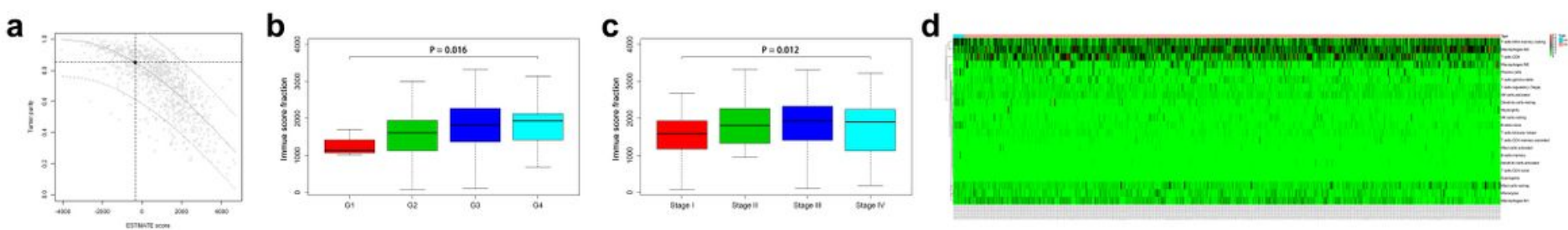
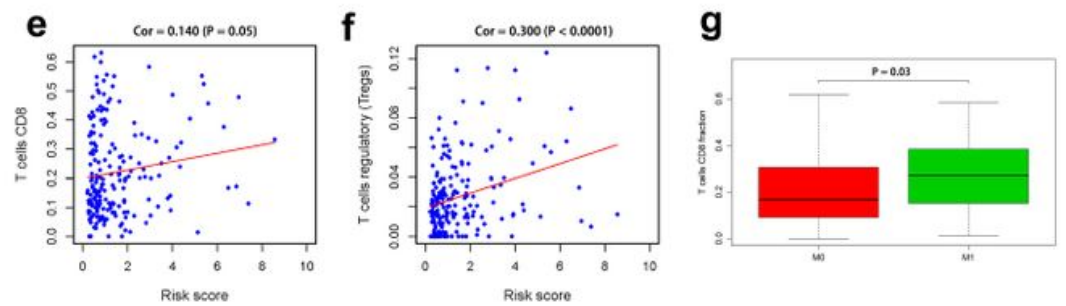

h
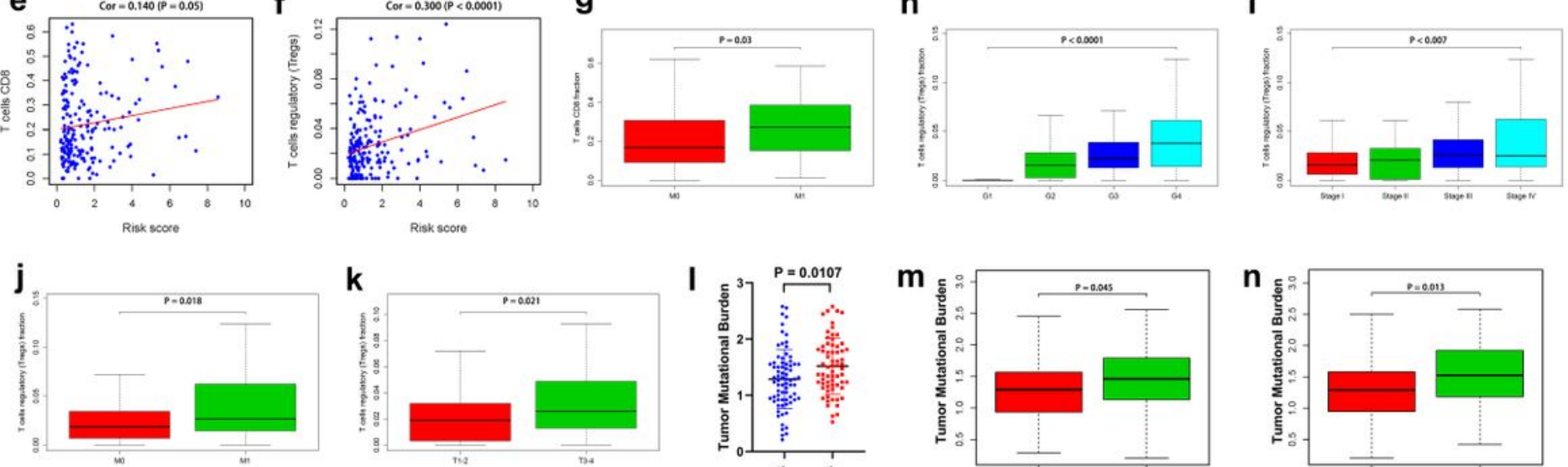

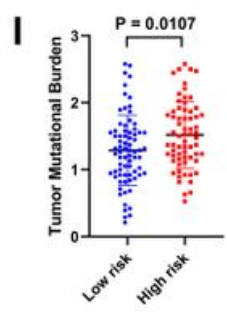

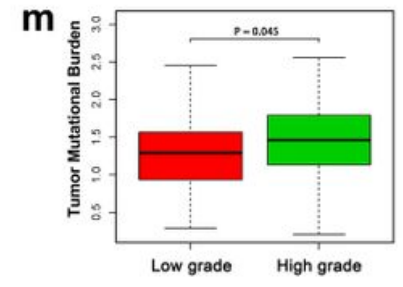

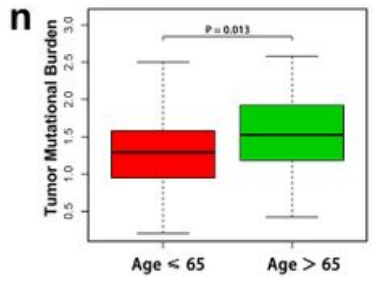




\section{Figure 3}

Relationship between present signature and tumor immune microenvironment. (a) Tumor purity assessed with ESTIMATE algorithm. (b) Immune score and deteriorated histological grade. (c) Immune score and advanced pathological stage. (d) TIICs assessed with CIBERSORT algorithm. (e) Correlation between risk score and CD8+ T cells. (f) Correlation between risk score and Tregs. (g) CD8+ T cells fraction and RCC metastasis. (h) Tregs fraction and deteriorated histological grade. (i) Tregs fraction and advanced pathological stage. (j) Tregs fraction and RCC metastasis. (k) Tregs fraction and tumor size. (l) TMB increased in high-risk group. (m) TMB and deteriorated histological grade. (n) TMB increased with age. P $<0.05$ was considered statistically significant.
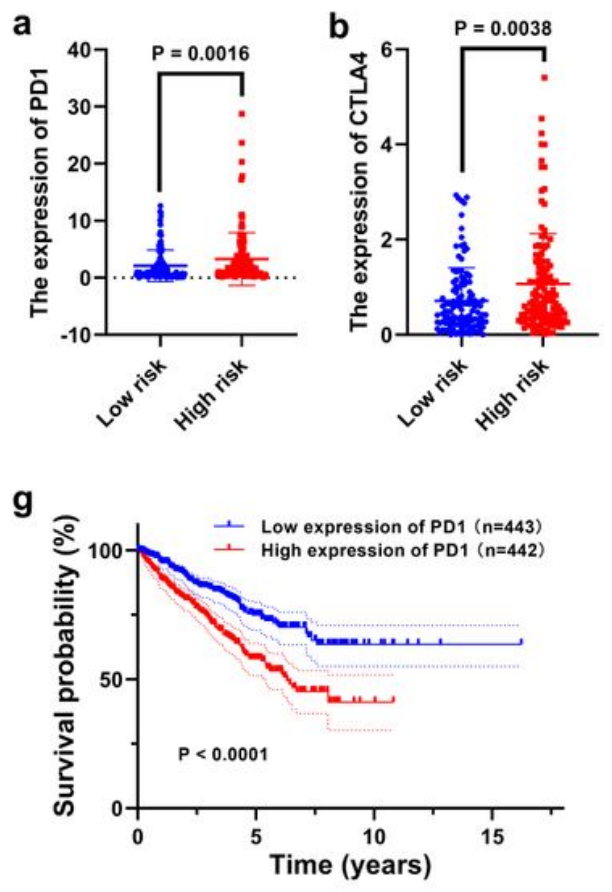
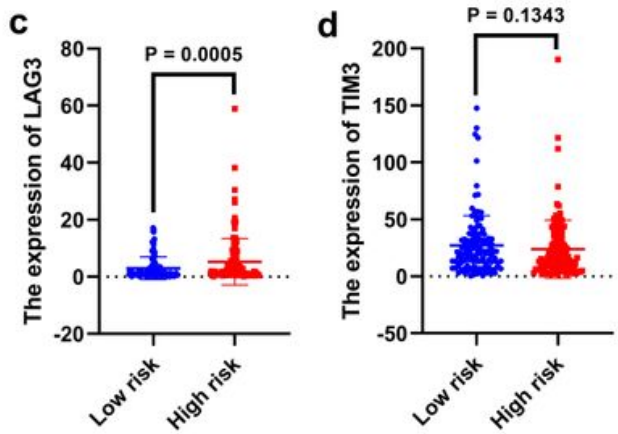

h

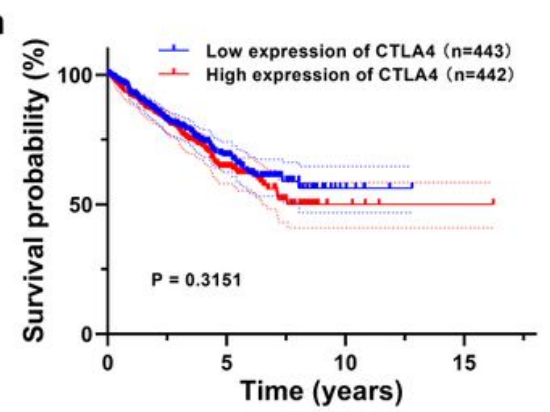

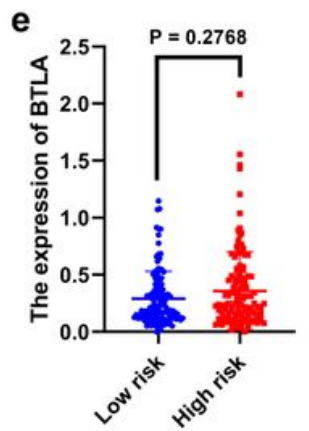

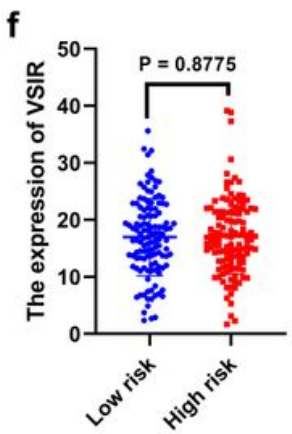

i

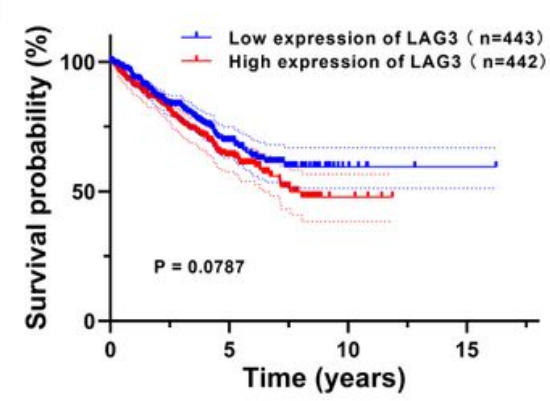

Figure 4

Relationship between present signature and immune checkpoints. Expression analysis of PD1 (a), CTLA4 (b), LAG3 (c), TIM3 (d), BTLA (e), and VSIR (f) between two risk groups. Kaplan-Meier curve of PD1 (g), CTLA4 (h), and LAG3 (i). Median expression level was used as cut-off value. $\mathrm{P}<0.05$ was considered statistically significant. 

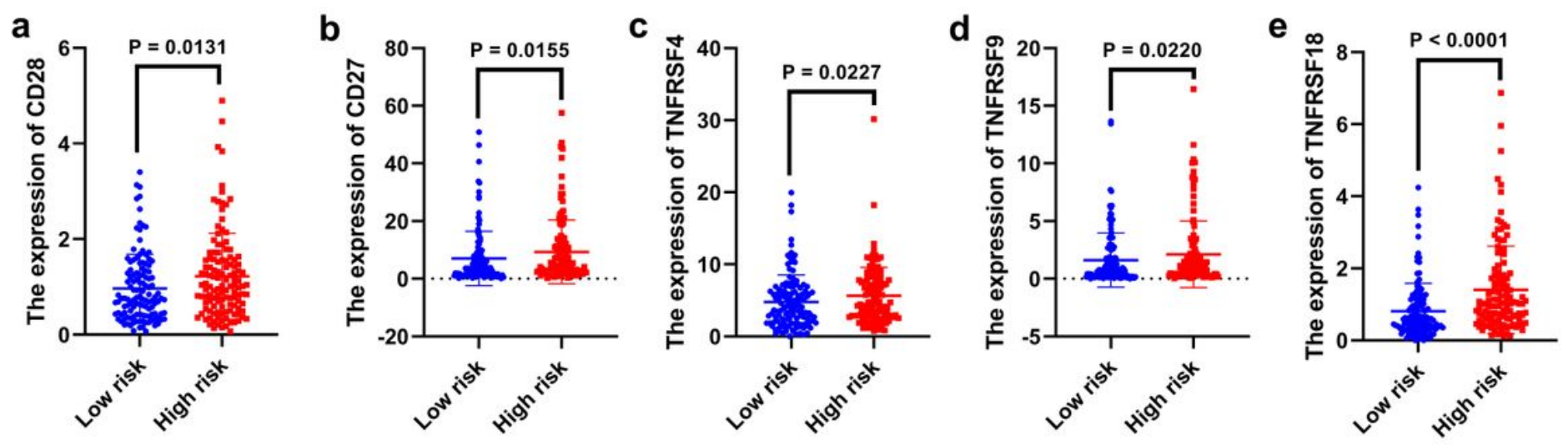

Figure 5

Relationship between present signature and immunostimulatory molecules. Expression analysis of CD28 (a), CD27 (b), TNFRSF4 (c), TNFRSF9 (d), and TNFRSF18 (e) between two risk groups. $P<0.05$ was considered statistically significant.
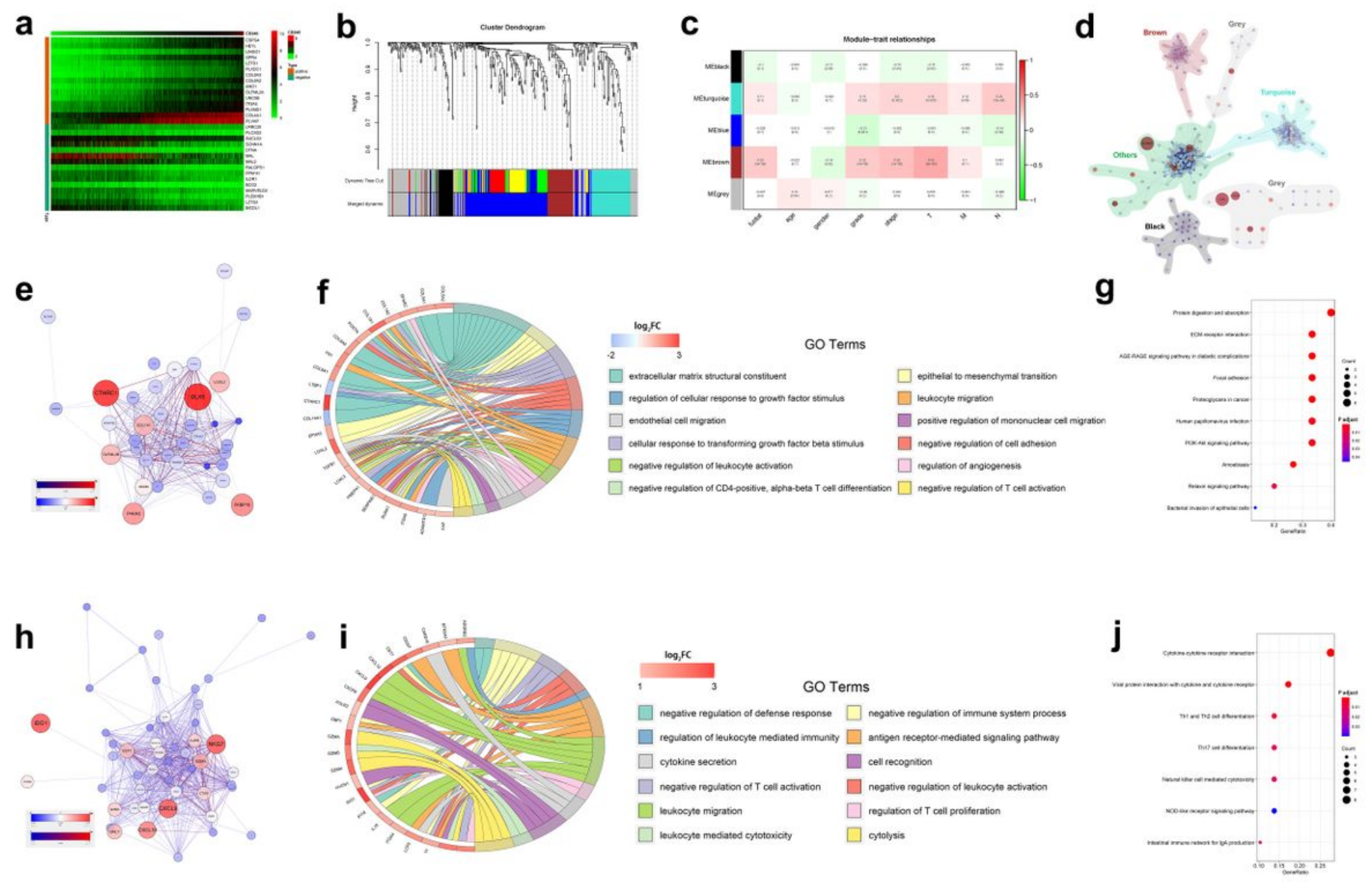

Figure 6 
WGCNA and enrichment analysis of CD248 co-expressed genes. (a) Co-expressed heatmap of CD248. (b) Identification of co-expressed modules. (c) Module-trait heatmap. (d) Interaction among co-expressed modules. Co-expressed network (e), GO enrichment analysis (f), and KEGG enrichment analysis (g) of brown modules. Co-expressed network (h), GO enrichment analysis (i), and KEGG enrichment analysis (j) of turquoise modules. FDR $<0.05$ was considered as statistically significant. 